\title{
Improving phlebotomy handover to doctors: a quality improvement project
}

Genevieve Shouls, Zakariya Jarrar, John Wickenden

St George's Hospital

\begin{abstract}
AIM: To design a hospital-standardised phlebotomy handover method to improve the communication between phlebotomists and doctors. To reduce delays in patient management and discharges which occur due to poor handover.

METHOD: Qualitative data was collected to gauge junior doctors' experiences of the current handover process. Quantitative data was collected over a two-week period across two medical wards to measure the proportion of requested bloods that could not be taken by phlebotomists that were successfully handed over to doctors. Brainstorming sessions were held with junior doctors, phlebotomists and ward staff in order to design a, cheap, effective, sustainable, hospital-wide method of handover. The chosen intervention was a red ward-based phlebotomy handover folder for phlebotomists to place stickers of unbled patients in. The folder was trialled on two medical wards. Feedback obtained helped improve the intervention before implementing it hospital-wide.
\end{abstract}

RESULTS: Seventeen of 23 junior doctors (74\%) felt that a formalised handover process would be very useful. Baseline measurement over two weeks revealed that $24 / 129$ blood tests ordered for phlebotomists to take were not taken. Only three (13\%) of these were handed over to doctors. Post-intervention, 18/106 blood tests requested were not taken. All $18(100 \%)$ were successfully handed over to doctors.

CONCLUSIONS: Implementation of a hospital-standardised phlebotomy handover folder dramatically improved the communication and handover between phlebotomists and doctors allowing for medical teams to take prompt action on unbled patients. This intervention will help improve patient safety, reduce delays in management/discharge and reduce the number of jobs handed over to evening on-call teams.

\section{Problem}

The inpatient phlebotomy service at St George's Hospital, London operates seven days a week throughout the year. Blood tests are an integral part of the day-to-day investigation and management of inpatients and the phlebotomists provide an invaluable service. Early morning blood tests mean they can be reviewed by midday, giving plenty of time for doctors to act on the results.

Understandably there are some patients that cannot be bled for particular reasons. For example, some patients are difficult to bleed, are absent from the bedside, or refuse to have blood taken. In such situations, it is vital that the relevant team is informed so that they can arrange for the blood to be taken and amend any management. Figure 1 summarises the current sequence of events that take place when a blood test is requested.

After starting work at St George's Hospital all three authors felt that the handover of untaken bloods between healthcare professionals was haphazard. There was great variability in the methods and success of handover between different wards. Only an informal system existed whereby phlebotomists either left blood stickers in various locations on wards, or handed them over to a member of the healthcare team, such as a nurse. Often stickers were incidentally found in numerous locations across the wards; on computer screens, on white boards, in the nursing handover book, on bedside tables, and on desks or telephones. These were usually not dated, making it difficult to figure out when they were meant to have been taken. Sometimes stickers weren't found at all meaning that the clinician wasn't aware of the failed blood request until they came to review the absent results later in the day.

Finding out that a blood test has not been done when reviewing blood tests (12-4pm), rather than much earlier in the day when the phlebotomist is on the ward (7-11am) causes huge problems for the medical and nursing team, therapists, and especially the patient.

A delay in blood results can cause:

1. Delayed discharges for patients waiting for final blood test results (warfarin levels, potassium levels, reducing CRP)

2. Delay in treatment of unwell patients

3. Over and under dosing of antibiotics that rely on therapeutic levels (eg gentamicin)

4. Delayed/cancelled surgery when there is insufficient time to correct blood test abnormalities

5. Asking the on-call team to review blood results for a patient that they don't know

All delays lead to compromised patient safety, bed blocking and inefficient use of NHS resources.

We set out to improve the phlebotomy handover method, to make sure that not only were all untaken blood tests were handed over to the medical team, but that this was done in a timely manner.

\section{Background}

To understand the scale of this handover problem around the 
hospital, we surveyed a total of 23 junior doctors. The survey suggested that on medical and surgical wards on average $45 \%$ and $51 \%$ of bloods that weren't taken were not handed over respectively. Of the junior doctors surveyed, $74 \%$ thought that formalising phlebotomy handover would be "very" useful. It is unclear whether anyone has tried to solve this problem in our hospital the past. The conversion of handwritten to computerised blood forms in recent years means that the blood taking and result reporting process has changed dramatically.

A team on the paediatric ward at Guys and St Thomas' Hospital also found problems with their phlebotomy handover method, and implemented a quality improvement project in July 2013. This showed a dramatic improvement in the handover of unsuccessful blood requests with a projected saving of $£ 6240$. Discussion with medical colleagues also suggests that standardised phlebotomy handover systems exist in other hospitals and that such a system would benefit St George's Hospital.

We met with the phlebotomy teams to gain a better understanding of how the process worked and worked with them to identify the point in the process that was best suited to an intervention. Before progressing any further we wanted to quantify the scale of the problem on two wards.

See supplementary file: ds3842.jpg - "Figure 1."

\section{Baseline measurement}

Data was collected over one week period on two medical wards in the hospital; a respiratory and an elderly care ward. We used a proforma to establish the number of blood tests ordered each day within the one week period, the number of these requests that were unsuccessful, and the number of unsuccessful requests that were handed over.

Between the two wards a total of 129 blood tests were ordered for the inpatient phlebotomy service, of these 24 bloods tests could not be taken (19\%), and only three of these 24 were handed over, meaning that only $13 \%$ of untaken bloods test were handed over to the doctors. This was greater than the estimates given from the junior doctors. It was clear from this data collection that a new handover method needed to be designed and implemented.

\section{Design}

The aim of the project was to design a standardised phlebotomy handover method. We explored the current process behind phlebotomy handover with junior doctors and the phlebotomy managers. This highlighted the problematic point in the process map allowing us to target our intervention appropriately: Firstly, no single formal handover method existed. Secondly, when a phlebotomist handed over to nurses there were occasions when this didn't get passed on, and thirdly there was no method of establishing the date of stickers left on the ward. We needed an intervention that addressed all three of these issues.
The initial meeting with the phlebotomy manager helped establish that a standardised method would be useful for both doctors and phlebotomists. We then consulted with nurses and matrons on the ward and they felt it important that the intervention ensured confidentiality, and that there was also a designated handover location. The intervention used must also be sustainable and easily reproducible. We considered a number of options, including pinboards, diaries, wallets attached to the wall, and folders. Of these interventions the diary seemed most appropriate: this addressed all three issues with the current process map and ensured confidentiality of patient details. However, after a second consultation with the phlebotomy manager it became clear that this wasn't suitable; diaries for all wards would be both expensive and impractical, requiring replacement and adaptation on a yearly basis. We therefore agreed on a red ringbinder with a polywallet inside for blood stickers to be left in. The phlebotomy manager also recommended that this folder also be used as a way for doctors to hand over paper blood test requests - to be used only in the event of the online ordering system going down. Therefore a second pollywallet was introduced into the folder for the downtime requests. They also suggested that the folder could be where doctors could put "Group \& save (G\&S)" paper request forms, and therefore it was agreed that the phlebotomists would check the folder for G\&S forms at the start of their ward round. We planned that the handover folder would be kept at the nursing desk on each ward.

The folder is a cheap, easy to implement intervention that ensures confidentiality of patient information on blood test stickers. They are sustainable and durable and can easily be stored on the wards. We planned to trial it on two wards for the first week and then roll it out across the rest of the wards.

\section{Strategy}

PDSA cycle 1: In collaboration with the phlebotomy manager the red ring binder was placed on two wards. This was trialled over one week. Despite the phlebotomy manager informing those phlebotomists scheduled to work on that ward about the folders, it quickly became clear that the folders weren't being used. The primary reason for this was the rotation of phlebotomists on the wards. A different phlebotomist worked on the ward each day and not all phlebotomists had been briefed about the intervention. As a result some phlebotomists were unaware of the need to use the folders. Of those that were using it, the main problem noticed was that the date of the blood test stickers was infrequently recorded.

PDSA cycle 2: In order to ensure all phlebotomists were aware of the folder we returned to the phlebotomy manager and attended two phlebotomy meetings, where we presented our handover folder to all inpatient phlebotomists employed by the hospital. We emphasised the handover process including the importance of dating the untaken blood test stickers. The phlebotomist gave us good feedback about the folders, and understood how they were to be used. We agreed to roll out of the folders across all of the wards to avoid confusion as to where the intervention was being implemented. The folders weren't appropriate for use on AMU given the unique nature of the department. Prior to roll out of the folders on a Monday morning, all junior doctors were emailed to inform 
them about the use of the handover folder, and that this was where they should look for untaken blood test stickers. The ward matrons and sisters were also emailed, and the phlebotomy manager also informed ward staff about the folder when placing them on the wards.

PDSA cycle 3: Following roll out of the folders across the hospital, we gained verbal feedback from our nursing and medical colleagues. Some highlighted that they could not find the folder on the ward, and we were quickly able to direct them to where it would be. Some wards, instead of keeping the folder at the nursing desk, kept the folder in a slot that was still present from when a folder was used to handover paper phlebotomy requests. On these wards we felt it appropriate to continue using the handover location, as the phlebotomists and ward staff were all aware about its presence and location. Three weeks after roll out we had good feedback with no further issues raised, and it was reported by ward staff that the folder was working well. No further changes were made.

See supplementary file: ds4174.jpg - "Before and after"

\section{Post-measurement}

After introduction of the phlebotomy handover folder onto the wards, data was collected for a further week on the same wards as pre-intervention. A total of 106 blood tests were ordered for the phlebotomy service. 18 (19\%) of these were not taken, and all 18 were handed over, meaning that $100 \%$ of untaken bloods test were handed over to the doctors. For all tests handed over it was clear what date the stickers were for. Graph 1 shows the percentage of successful handovers pre and post-intervention.

See supplementary file: ds4177.gif - "Graph 1"

\section{Lessons and limitations}

During this project we have learnt that to keep this intervention sustainable there must be a named project ambassador. This person can continue overseeing the effective use of the handover folders, especially once the initiating junior doctors rotate hospitals. We are very fortunate that the hospital's phlebotomy manager, who has been so involved in both the design and implementation of the project, is willing to act as the project ambassador, to continue encouraging the effective use of this handover folder in the future.

The very large number of people involved in the project made coordination and communication between all parties difficult at times. Similarly ensuring that the design of the intervention was satisfactory to nurses, phlebotomists and doctors was a demanding task. As a result the project took longer than expected and was implemented over the course of junior doctor rotations. This made it difficult to reinforce the use of folders on particular wards. If repeating a project in future it may be wiser to implement it at the start of junior doctor placements.

A limitation highlighted by the use of the intervention on the wards was that surgical teams, who aren't based on a single ward but have outlying patients across the hospital found it impractical to check every ward folder. A solution to this could be that a nominated member of staff on each ward hand over stickers to the relevant team when they arrive on the ward.

There is a potential issue with the correct dating of stickers - we are relying on phlebotomists to remove old stickers from the wallet before replacing them with new ones with the date on them. Although this process was working well at the time of re-audit, there is potential for errors to occur making it difficult to date the stickers. This can be avoided by future reinforcement of the handover process to the phlebotomists by the phlebotomy manager; the sustainability of this aspect should be re-audited at a later date.

\section{Conclusion}

Recent changes in healthcare working patterns have necessitated effective handover to ensure patient safety. The World Health Organisation and many studies have identified standardised handover methods as a crucial factor in ensuring patient safety and the provision of excellent care and this project aimed to improve just one such process. It was clear from both informal discussions with colleagues and formal data collection that a problem existed with phlebotomy handover. The handover of only $13 \%$ of untaken bloods could have a considerable impact on both patient safety and NHS resources. With a post-intervention handover of $100 \%$ this project has demonstrably resolved that issue and if this project could prevent just one delayed discharge per day across the wards of St George's Hospital then this could have a potential saving of £99645 per annum for the trust. Furthermore the simplicity and low cost of the intervention means that it is easily sustainable, especially given the phlebotomy manager has taken on the responsibility of keeping the project going in the future.

\section{References}

World Health Organisation, Communication During Patient Handovers. Patient Safety Solutions May 2007 1(3)

BMA, Safe handover: safe patients. Guidance on clinical handover for clinicians and managers. Available at http://bma.org.uk/-/media/files/pdfs/practical\%20advice\%20at\%20w ork/contracts/safe\%20handover\%20safe\%20patients.pdf. [Accessed 23.05.2014]

Jeffcott SA, Ibrahim JE, Cameron PA. Resilience in healthcare and clinical handover. Qual Saf Health Care. 2009 Aug;18(4):256-60

NHS, Reference costs 2012 to 2013. Available https://www.gov.uk/government/uploads/system/uploads/attachmen t data/file/261154/nhs reference costs 2012-13 acc.pdf [Accessed 31.05.14]

\section{Declaration of interests}




\section{Acknowledgements}

Dianne Campbell - phlebotomy manager

The phlebotomy team 